\title{
Statistical Theory of Certain Distribution Functions in MHD Turbulent Flow for Velocity and Concentration Undergoing A First Order Reaction in a Rotating System
}

\author{
M. A. K. Azad*, M. A. Aziz and M.S.Alam Sarker \\ Department of Applied Mathematics, University of Rajshahi-6205, Bangladesh.
}

\begin{abstract}
In this paper, the distribution functions for simultaneous velocity, magnetic, temperature, concentration fields and reaction in MHD turbulent flow undergoing a first order reaction in a rotating system is studied. Finally, the transport equations for evolution of distribution functions have been derived. The various properties of the distribution function have been discussed. The obtained one-point equation is compared with the first equation of BBGKY hierarchy of equations in the kinetic theory of gases.
\end{abstract}

Keywords: Distribution functions, MHD Turbulence, Concentration, First order reaction, Rotating system

\section{Introduction}

The kinetic theory of gases and the statistical theory of fluid mechanics are the two major and distinct areas of investigations in statistical mechanics. In the past, several authors discussed the distribution functions in the statistical theory of turbulence. (Lundgren, 1967) derived a hierarchy of coupled equations for multi-point turbulence velocity distribution functions, which resemble with BBGKY hierarchy of equations of (Ta-Yu-Wu, 1966) in the kinetic theory of gasses. (Kishore,1978) studied the distribution functions in the statistical theory of MHD turbulence of an incompressible fluid. (Pope, 1981b) derived the transport equation for the joint probability density function of velocity and scalars in turbulent flow. (Kishore and Singh, 1984) derived the transport equation for the bivariate joint distribution function of velocity and temperature in turbulent flow. Also (Kishore and Singh, 1985) have been derived the transport equation for the joint distribution function of velocity, temperature and concentration in convective turbulent flow. (Dixit and Upadhyay,1989b) considered the distribution functions in the statistical theory of MHD turbulence of an incompressible fluid in the presence of the coriolis force. (Kollman and Janicka,1982) derived the transport equation for the probability density function of a scalar in turbulent shear flow and considered a closure model based on gradient -flux model.But at this stage, one is met with the difficulty that the $\mathrm{N}$-point distribution function depends upon the $\mathrm{N}+1$-point

\footnotetext{
* Corresponding author: E-mail: aazizphd@gmail.com
}

distribution function and thus result is an unclosed system. This so-called "closer problem" is encountered in turbulence, kinetic theory and other non-linear system. (Sarker and Kishore, 1991a) discussed the distribution functions in the statistical theory of convective MHD turbulence of an incompressible fluid. Also (Sarker and Kishore, 1999) studied the distribution functions in the statistical theory of convective MHD turbulence of mixture of a miscible incompressible fluid. (Sarker and Islam, 2002) studied the Distribution functions in the statistical theory of convective MHD turbulence of an incompressible fluid in a rotating system. (Islam and Sarker, 2007) also studied Distribution functions in the statistical theory of MHD turbulence for velocity and concentration undergoing a first order reaction.

In this paper, we have studied the statistical theory of distribution functions for simultaneous velocity, magnetic, temperature, concentration fields and reaction in MHD turbulent flow undergoing a first order reaction in a rotating system. Finally, the transport equations for evolution of distribution functions have been derived and various properties of the distribution function have been discussed. The resulting onepoint equation is compared with the first equation of BBGKY hierarchy of equations and the closure difficulty is to be removed as in the case of ordinary turbulence. 


\section{Basic Equations}

The equations of motion and continuity for viscous incompressible dusty fluid MHD turbulent flow, the diffusion equations for the temperature and concentration undergoing a first order chemical reaction in a rotating system are given by

$$
\begin{aligned}
& \frac{\partial u_{\alpha}}{\partial t}+\frac{\partial}{\partial x_{\beta}}\left(u_{\alpha} u_{\beta}-h_{\alpha} h_{\beta}\right)=-\frac{\partial w}{\partial x_{\alpha}}+ \\
& \nu \nabla^{2} u_{\alpha}-2 \epsilon_{m \alpha \beta} \Omega_{m} u_{\alpha} \\
& \frac{\partial h_{\alpha}}{\partial t}+\frac{\partial}{\partial x_{\beta}}\left(h_{\alpha} u_{\beta}-u_{\alpha} h_{\beta}\right)=\lambda \nabla^{2} h_{\alpha}
\end{aligned}
$$

$\frac{\partial \theta}{\partial t}+u_{\beta} \frac{\partial \theta}{\partial x_{\beta}}=\gamma \nabla^{2} \theta$

$\frac{\partial C}{\partial t}+u_{\beta} \frac{\partial C}{\partial x_{\beta}}=D \nabla^{2} C-R C$

with $\frac{\partial u_{\alpha}}{\partial x_{\alpha}}=\frac{\partial v_{\alpha}}{\partial x_{\alpha}}=\frac{\partial h_{\alpha}}{\partial x_{\alpha}}=0$

where

$u_{\alpha}(x, t), \alpha$ - component of turbulent velocity; $\mathrm{h}_{\alpha}(x, t)$, $\alpha$ - component of magnetic field $\theta(x, t)$, temperature fluctuation; $\mathrm{C}$, concentration of contaminants; $\mathrm{v}_{\alpha}$, dust particle velocity; $\mathrm{R}$, specific reaction rate; $\in_{m \alpha \beta}$, alternating tensor; $\mathrm{N}$, constant number of density of the dust particle;

$w(\hat{x}, t)=P / \rho+\frac{1}{2}|h|^{2}+\frac{1}{2}|\hat{\Omega} \times \hat{x}|^{2}$, total pressure $P(\hat{x}, t)$

hydrodynamic pressure $\rho$, fluid density; $\Omega$, angular velocity of a uniform rotation; $v$, Kinetic viscosity $\lambda=(4 \pi \mu \sigma)^{-1}$ magnetic diffusivity; $\gamma=\frac{k_{T}}{\rho c_{p}}$ thermal diffusivity; $\mathrm{c}_{\mathrm{p}}$, specific heat at constant pressure; $\mathrm{k}_{\mathrm{T}}$, thermal conductivity; $\sigma$, electrical conductivity; $\mu$, magnetic permeability; D, diffusive co-efficient for contaminants.

The repeated suffices are assumed over the values 1, 2 and 3 and unrepeated suffices may take any of these values. Here $\mathrm{u}, \mathrm{h}$ and $\mathrm{x}$ are vector quantities in the whole process.
The total pressure $\mathrm{w}$ which, occurs in equation (1) may be eliminated with the help of the equation obtained by taking the divergence of equation (1)

$$
\begin{aligned}
& \nabla^{2} w=-\frac{\partial^{2}}{\partial x_{\alpha} \partial x_{\beta}}\left(u_{\alpha} u_{\beta}-h_{\alpha} h_{\beta}\right)= \\
& -\left[\frac{\partial u_{\alpha}}{\partial x_{\beta}} \frac{\partial u_{\beta}}{\partial x_{\alpha}}-\frac{\partial h_{\alpha}}{\partial x_{\beta}} \frac{\partial h_{\beta}}{\partial x_{\alpha}}\right] .
\end{aligned}
$$

In a conducting infinite fluid only the particular solution of the Equation (6) is related, so that

$$
w=\frac{1}{4 \pi} \int\left[\frac{\partial u_{\alpha}^{\prime}}{\partial x_{\beta}^{\prime}} \frac{\partial u_{\beta}^{\prime}}{\partial x_{\alpha}^{\prime}}-\frac{\partial h_{\alpha}^{\prime}}{\partial x_{\beta}^{\prime}} \frac{\partial h_{\beta}^{\prime}}{\partial x_{\alpha}^{\prime}}\right] \frac{\partial \bar{x}^{\prime}}{\left|\bar{x}^{\prime}-\bar{x}\right|}
$$

Hence equation (1) - (4) becomes

$$
\begin{aligned}
& \frac{\partial u_{\alpha}}{\partial t}+\frac{\partial}{\partial x_{\beta}}\left(u_{\alpha} u_{\beta}-h_{\alpha} h_{\beta}\right)=-\frac{1}{4 \pi} \frac{\partial}{\partial x_{\alpha}} \int \\
& {\left[\frac{\partial u_{\alpha}^{\prime}}{\partial x_{\beta}^{\prime}} \frac{\partial u_{\beta}^{\prime}}{\partial x_{\alpha}^{\prime}}-\frac{\partial h_{\alpha}^{\prime}}{\partial x_{\beta}^{\prime}} \frac{\partial h_{\beta}^{\prime}}{\partial x_{\alpha}^{\prime}}\right] \frac{d \bar{x}^{\prime}}{\left|\bar{x}^{\prime}-\bar{x}\right|}+\nu \nabla^{2} u_{\alpha}} \\
& -2 \in \in_{m \alpha \beta} \Omega_{m} u_{\alpha}
\end{aligned}
$$

$\frac{\partial h_{\alpha}}{\partial t}+\frac{\partial}{\partial x_{\beta}}\left(h_{\alpha} u_{\beta}-u_{\alpha} h_{\beta}\right)=\lambda \nabla^{2} h_{\alpha}$

$\frac{\partial \theta}{\partial t}+u_{\beta} \frac{\partial \theta}{\partial x_{\beta}}=\gamma \nabla^{2} \theta$

$\frac{\partial C}{\partial t}+u_{\beta} \frac{\partial C}{\partial x_{\beta}}=D \nabla^{2} C-R C$

\section{Formulation of the Problem}

We consider the turbulence and the concentration fields are homogeneous, the chemical reaction and the local mass transfer have no effect on the velocity field and the reaction rate and the diffusivity are constant. We also consider a large ensemble of identical fluids in which each member is an infinite incompressible reacting and heat conducting fluid in turbulent state. The fluid velocity u, Alfven velocity h, temperature $\theta$ and concentration $\mathrm{C}$, are randomly distributed functions of position and time and satisfy their field. Different members of ensemble are subjected to different initial conditions and our aim is to find out a way by which we can deter- 
mine the ensemble averages at the initial time. Certain microscopic properties of conducting fluids, such as total energy, total pressure, stress tensor which are nothing but ensemble averages at a particular time, can be determined with the help of the bivariate distribution functions (defined as the averaged distribution functions with the help of Dirac delta-functions). Our present aim is to construct the distribution functions, study its properties and derive an equation for its evolution of this distribution functions.

\section{* Distribution Function in MHD Turbulence and Their Properties}

In MHD turbulence, we may consider the fluid velocity $\mathrm{u}$, Alfven velocity $\mathrm{h}$, temperature $\theta$, concentration $\mathrm{C}$ and constant reaction rate $\mathrm{R}$ at each point of the flow field. (Lundgren,1967) has studied the flow field on the basis of one variable character only (namely the fluid $u$ ), but we can study it for two or more variable characters as well. The corresponding to each point of the flow field, we have four measurable characteristics. We represent the four variables by v, g, $\phi$ and $\psi$ and denote the pairs of these variables at the points $\bar{x}^{(1)}, \bar{x}^{(2)},-----, \bar{x}^{(n)}$ as $\left(\bar{v}^{(1)}, \bar{g}^{(1)}, \phi^{(1)}, \psi^{(1)}\right)$, $\left(\bar{v}^{(2)}, \bar{g}^{(2)}, \phi^{(2)}, \psi^{(2)}\right),------\left(\bar{v}^{(n)}, \bar{g}^{(n)}, \phi^{(n)}, \psi^{(n)}\right)$ at a fixed instant of time. It is possible that the same pair may be occur more than once; therefore, we simplify the problem by an assumption that the distribution is discrete (in the sense that no pairs occur more than once). Symbolically we can express the distribution as

$$
\begin{aligned}
& \left\{\left(\bar{v}^{(1)}, \bar{g}^{(1)}, \phi^{(1)}, \psi^{(1)}\right),\left(\bar{v}^{(2)}, \bar{g}^{(2)}, \phi^{(2)}, \psi^{(2)}\right) ;\right. \\
& \left.------\left(\bar{v}^{(n)}, \bar{g}^{(n)}, \phi^{(n)}, \psi^{(n)}\right)\right\} .
\end{aligned}
$$

Instead of considering discrete points in the flow field, if we consider the continuous distribution of the variables $\bar{v}, \bar{g}, \phi$ and $\psi$ over the entire flow field, statistically behavior of the fluid may be described by the distribution function $F(\bar{v}, \bar{g}$, $\phi, \psi)$ which is normalized so that

$$
\int F(\bar{v}, \bar{g}, \phi, \psi) d \bar{v}, d \bar{g} d \phi d \psi=1
$$

where the integration ranges over all the possible values of $\mathrm{v}$, $\mathrm{g}, \phi$ and $\psi$. We shall make use of the same normalization condition for the discrete distributions also. The distribution functions of the above quantities can be defined in terms of Dirac delta functions.
The one-point distribution function $F_{1}^{(1)}\left(v^{(1)}, g^{(1)}, \phi^{(1)}, \psi^{(1)}\right)$ defined so that $F_{1}^{(1)}\left(v^{(1)}, g^{(1)}, \phi^{(1)}, \psi^{(1)}\right) d v^{(1)} d g^{(1)} d \phi^{(1)} d \psi^{(1)}$ is the probability that the fluid velocity, Alfven velocity, temperature and concentration field at a time $t$ are in the element $d v^{(1)}$ about $v^{(1)}, \mathrm{dg}^{(1)}$ about $\mathrm{g}^{(1)}, \mathrm{d} \phi^{(1)}$ about $\phi^{(1)}$ and $\mathrm{d} \psi^{(1)}$ about $\psi^{(1)}$ respectively and is given by

$$
\begin{aligned}
& F_{1}^{(1)}\left(v^{(1)}, g^{(1)}, \phi^{(1)}, \psi^{(1)}\right)=\left\langle\delta\left(u^{(1)}-v^{(1)}\right) \delta\left(h^{(1)}-g^{(1)}\right)\right. \\
& \left.\delta\left(\theta^{(1)}-\phi^{(1)}\right) \delta\left(C^{(1)}-\psi^{(1)}\right)\right\rangle
\end{aligned}
$$

where $\delta$ is the Dirac delta-function defined as

$\int \delta(\bar{u}-\bar{v}) d \bar{v}= \begin{cases}1 & \text { at the point } \bar{u}=\bar{v} \\ 0 & \text { elsewhere }\end{cases}$

Two-point distribution function is given by

$$
\begin{aligned}
& F_{2}^{(1,2)}=\left\langle\delta\left(u^{(1)}-v^{(1)}\right) \delta\left(h^{(1)}-g^{(1)}\right) \delta\left(\theta^{(1)}-\phi^{(1)}\right)\right. \\
& \delta\left(C^{(1)}-\psi^{(1)}\right) \delta\left(u^{(2)}-v^{(2)}\right) \delta\left(h^{(2)}-g^{(2)}\right) \\
& \left.\delta\left(\theta^{(2)}-\phi^{(2)}\right) \delta\left(C^{(2)}-\psi^{(2)}\right)\right\rangle
\end{aligned}
$$

and three point distribution function is given by

$$
\begin{aligned}
& F_{3}^{(1,2,3)}=\left\langle\delta ( u ^ { ( 1 ) } - v ^ { ( 1 ) } ) \delta ( h ^ { ( 1 ) } - g ^ { ( 1 ) } ) \delta ( \theta ^ { ( 1 ) } - \phi ^ { ( 1 ) } ) \delta \left( C^{(1)}\right.\right. \\
& \left.-\psi^{(1)}\right) \delta\left(u^{(2)}-v^{(2)}\right) \delta\left(h^{(2)}-g^{(2)}\right) \\
& \times \delta\left(\theta^{(2)}-\phi^{(2)}\right) \delta\left(C^{(2)}-\psi^{(2)}\right) \delta\left(u^{(3)}-v^{(3)}\right) \delta\left(h^{(3)}-g^{(3)}\right) \\
& \left.\delta\left(\theta^{(3)}-\phi^{(3)}\right) \delta\left(C^{(3)}-\psi^{(3)}\right)\right\rangle
\end{aligned}
$$

Similarly, we can define an infinite numbers of multi-point distribution functions $\mathrm{F}_{4}^{(1,2,3,4)}, \mathrm{F}_{5}^{(1,2,3,4,5)}$ and so on.

The distribution functions so constructed have the following properties:

\section{(A) Reduction Properties}

Integration with respect to pair of variables at one-point, lowers the order of distribution function by one. For example,

$$
\begin{aligned}
& \iiint \int F_{1}^{(1)} d v^{(1)} d g^{(1)} d \phi^{(1)} d \psi^{(1)}=1, \\
& \iiint \int F_{2}^{(1,2)} d v^{(2)} d g^{(2)} d \phi^{(2)} d \psi^{(2)}=F_{1}^{(1)}, \\
& \iiint \int F_{3}^{(1,2,3)} d v^{(3)} d g^{(3)} d \phi^{(3)} d \psi^{(3)}=F_{2}^{(1,2)} \text { etc. }
\end{aligned}
$$


Also the integration with respect to any one of the variables, reduces the number of Delta-functions from the distribution function by one as

$$
\begin{gathered}
\int F_{1}^{(1)} d v^{(1)}=\left\langle\delta\left(h^{(1)}-g^{(1)}\right) \delta\left(\theta^{(1)}-\phi^{(1)}\right) \delta\left(C^{(1)}-\psi^{(1)}\right)\right\rangle, \\
\int F_{1}^{(1)} d g^{(1)}=\left\langle\delta\left(u^{(1)}-v^{(1)}\right) \delta\left(\theta^{(1)}-\phi^{(1)}\right) \delta\left(C^{(1)}-\psi^{(1)}\right)\right\rangle, \\
\int F_{1}^{(1)} d \phi^{(1)}=\left\langle\delta\left(u^{(1)}-v^{(1)}\right) \delta\left(h^{(1)}-g^{(1)}\right) \delta\left(C^{(1)}-\psi^{(1)}\right)\right\rangle \\
\text { and } \int F_{2}^{(1,2)} d v^{(2)}=\left\langle\delta\left(u^{(1)}-v^{(1)}\right) \delta\left(h^{(1)}-g^{(1)}\right)\right. \\
\quad \delta\left(\theta^{(1)}-\phi^{(1)}\right) \delta\left(C^{(1)}-\psi^{(1)}\right) \delta\left(h^{(2)}-g^{(2)}\right) \\
\left.\delta\left(\theta^{(2)}-\phi^{(2)}\right) \delta\left(C^{(2)}-\psi^{(2)}\right)\right\rangle
\end{gathered}
$$

\section{(B) Separation Properties}

The pairs of variables at the two points are statistically independent of each other if these points are far apart from each other in the flow field i.e.,

$$
\operatorname{Lim}_{\left|\wp^{(2)} \rightarrow \bar{x}^{(1)}\right| \rightarrow \infty} F_{2}^{(1,2)}=F_{1}^{(1)} F_{1}^{(2)}
$$

and similarly, $\operatorname{Lim}_{\left|\bar{x}^{(3)} \rightarrow \bar{x}^{(2)}\right| \rightarrow \infty} F_{3}^{(1,2,3)}=F_{2}^{(1,2)} F_{1}^{(3)}$ etc

\section{(C) Co-incidence Property}

When two points coincide in the flow field, the components at these points should be obviously the same that is $F_{2}(1,2)$ must be zero. Thus $\bar{v}^{(2)}=\bar{v}^{(1)}, \quad g^{(2)}=g^{(1)}, \phi^{(2)}=\phi^{(1)}$ and $\psi^{(2)}=\psi^{(1)}$, but $\mathrm{F}_{1}^{(1,2)}$ must also have the property.

$$
\iiint \int F_{2}^{(1,2)} d v^{(2)} d g^{(2)} d \phi^{(2)} d \psi^{(2)}=F_{1}^{(1)}
$$

and hence it follows that

$$
\begin{aligned}
& \operatorname{Lim}_{\left|\bar{x}^{(3)} \rightarrow \bar{x}^{(2)}\right| \rightarrow \infty} \int F_{2}^{(1,2)}=F_{1}^{(1)} \delta\left(v^{(2)}-v^{(1)}\right) \\
& \delta\left(g^{(2)}-g^{(1)}\right) \delta\left(\phi^{(2)}-\phi^{(1)}\right) \delta\left(\psi^{(2)}-\psi^{(1)}\right)
\end{aligned}
$$

Similarly, $\operatorname{Lim}_{\left|\bar{x}^{(3)} \rightarrow \bar{x}^{(2)}\right| \rightarrow \infty} \int F_{3}^{(1,2,3)}=F_{2}^{(1,2)} \delta\left(v^{(3)}-v^{(1)}\right)$ $\delta\left(g^{(3)}-g^{(1)}\right) \delta\left(\phi^{(3)}-\phi^{(1)}\right) \delta\left(\psi^{(3)}-\psi^{(1)}\right)$ etc.

\section{(D) Symmetric Conditions}

$F_{n}^{(1,2, r,------s,-----n)}=F_{n}^{(1,2,-----s,---r,---n)}$

(E) Incompressibility Conditions

(i) $\iint \frac{\partial F_{n}^{(1,2,---n)}}{\partial x_{\alpha}^{(r)}} v_{\alpha}^{(r)} d \bar{v}^{(r)} d \bar{h}^{(r)}=0$,

(ii) $\iint \frac{\partial F_{n}^{(1,2,---n)}}{\partial x_{\alpha}^{(r)}} h_{\alpha}^{(r)} d \bar{v}^{(r)} d \bar{h}^{(r)}=0$.

\section{Continuity Equation in Terms of Distribution Functions}

An infinite number of continuity equations can be derived for the convective MHD turbulent flow and the continuity equations can be easily expressed in terms of distribution functions and are obtained directly by $\operatorname{div} u=0$. Taking ensemble average of equation (5)

$0=\left\langle\frac{\partial u_{\alpha}^{(1)}}{\partial x_{\alpha}^{(1)}}\right\rangle=\left\langle\frac{\partial}{\partial x_{\alpha}^{(1)}} u_{\alpha}^{(1)} \iiint \int F_{1}^{(1)} d v^{(1)} d g^{(1)} d \phi^{(1)} d \psi^{(1)}\right\rangle$

$=\frac{\partial}{\partial x_{\alpha}^{(1)}}\left\langle u_{\alpha}^{(1)} \iiint \int F_{1}^{(1)} d v^{(1)} d g^{(1)} d \phi^{(1)} d \psi^{(1)}\right\rangle$

$=\frac{\partial}{\partial x_{\alpha}^{(1)}} \iiint \int\left\langle u_{\alpha}^{(1)}\right\rangle\left\langle F_{1}^{(1)}\right\rangle d v^{(1)} d g^{(1)} d \phi^{(1)} d \psi^{(1)}$

$=\frac{\partial}{\partial x_{\alpha}^{(1)}} \iiint \int v_{\alpha}^{(1)} F_{1}^{(1)} d v^{(1)} d g^{(1)} d \phi^{(1)} d \psi^{(1)}$

$=\iiint \int \frac{\partial F_{1}^{(1)}}{\partial x_{\alpha}^{(1)}} v_{\alpha}^{(1)} d v^{(1)} d g^{(1)} d \phi^{(1)} d \psi^{(1)}$

and similarly,

$$
0=\iiint \int \frac{\partial F_{1}^{(1)}}{\partial x_{\alpha}^{(1)}} g_{\alpha}^{(1)} d v^{(1)} d g^{(1)} d \phi^{(1)} d \psi^{(1)}
$$

which are the first order continuity equations in which only one point distribution function is involved. For second-order 
continuity equations, if we multiply the continuity equation by

$\delta\left(u^{(2)}-v^{(2)}\right) \delta\left(h^{(2)}-g^{(2)}\right) \delta\left(\theta^{(2)}-\phi^{(2)}\right) \delta\left(C^{(2)}-\psi^{(2)}\right)$

and if we take the ensemble average, we obtain

$$
\begin{aligned}
o= & \left\langle\delta\left(u^{(2)}-v^{(2)}\right) \delta\left(h^{(2)}-g^{(2)}\right) \delta\left(\theta^{(2)}-\phi^{(2)}\right)\right. \\
& \left.\delta\left(C^{(2)}-\psi^{(2)}\right) \frac{\partial u_{\alpha}^{(1)}}{\partial x_{\sim}^{(1)}}\right\rangle \\
& =\frac{\partial}{\partial x_{\alpha}^{(1)}}\left\langle\delta\left(u^{(2)}-v^{(2)}\right) \delta\left(h^{(2)}-g^{(2)}\right)\right. \\
& \left.\delta\left(\theta^{(2)}-\phi^{(2)}\right) \delta\left(C^{(2)}-\psi^{(2)}\right) u_{\alpha}^{(1)}\right\rangle \\
& =\frac{\partial}{\partial x_{\alpha}^{(1)}} \int\left\langle u_{\alpha}^{(1)} \delta\left(u^{(1)}-v^{(1)}\right) \delta\left(h^{(1)}-g^{(1)}\right)\right. \\
& \delta\left(\theta^{(1)}-\phi^{(1)}\right) \delta\left(C^{(1)}-\psi^{(1)}\right) \\
& \times \delta\left(u^{(2)}-v^{(2)}\right) \delta\left(h^{(2)}-g^{(2)}\right) \delta\left(\theta^{(2)}-\phi^{(2)}\right) \\
& \left.\delta\left(C^{(2)}-\psi^{(2)}\right)\right\rangle \\
& o=\frac{\partial}{\partial x_{\alpha}^{(1)}} \iiint \int v_{\alpha}^{(1)} F_{2}^{(1,2)} d v^{(1)} d g^{(1)} d \phi^{(1)} d \psi^{(1)}
\end{aligned}
$$

and similarly,

$o=\frac{\partial}{\partial x_{\alpha}^{(1)}} \iiint \int g_{\alpha}^{(1)} F_{2}^{(1,2)} d v^{(1)} d g^{(1)} d \phi^{(1)} d \psi^{(1)}$

The Nth - order continuity equations are

$o=\frac{\partial}{\partial x_{\alpha}^{(1)}} \iiint \int v_{\alpha}^{(1)} F_{N}^{(1,2,---, N)} d v^{(1)} d g^{(1)} d \phi^{(1)} d \psi^{(1)}$

and

$o=\frac{\partial}{\partial x_{\alpha}^{(1)}} \iiint \int g_{\alpha}^{(1)} F_{N}^{(1,2, \ldots \ldots . ., N)} d v^{(1)} d g^{(1)} d \phi^{(1)} d \psi^{(1)}$

The continuity equations are symmetric in their arguments i.e.;

$$
\begin{aligned}
& \frac{\partial}{\partial x_{\alpha}^{(r)}} \iiint \int\left(v_{\alpha}^{(r)} F_{N}^{(1,2, \ldots \ldots . ., r . N)} d v^{(r)} d g^{(r)} d \phi^{(r)} d \psi^{(r)}\right) \\
& =\frac{\partial}{\partial x_{\alpha}^{(s)}} \iiint \int v_{\alpha}^{(s)} F_{N}^{(1,2, \ldots . ., r, s, \ldots . . N)} d v^{(s)} d g^{(s)} d \phi^{(s)} d \psi^{(s)}
\end{aligned}
$$

Since the divergence property is an important property and it is easily verified by the use of the property of distribution function as

$$
\begin{aligned}
& \frac{\partial}{\partial x_{\alpha}^{(1)}} \iiint \int v_{\alpha}^{(1)} F_{1}^{(1)} d v^{(1)} d g^{(1)} d \phi^{(1)} d \psi^{(1)} \\
& =\frac{\partial}{\partial x_{\alpha}^{(1)}}\left\langle u_{\alpha}^{(1)}\right\rangle=\left\langle\frac{\partial u_{\alpha}^{(1)}}{\partial x_{\alpha}(1)}\right\rangle=o
\end{aligned}
$$

and all the properties of the distribution function obtained in section (*) can also be verified.

\section{Equations for Evolution of Distribution Functions}

The equations (8)-(11) will be used to convert these into a set of equations for the variation of the distribution function with time. This, in fact, is done by making use of the definitions of the constructed distribution functions, differentiating them partially with respect to time, making some suitable operations on the right-hand side of the equation so obtained and lastly replacing the time derivative of $v, h, \theta$ and $\mathrm{C}$ from the equations (8)- (11). Differentiating equation (12), and then using equations (8)-(11) we get,

$$
\begin{aligned}
& \frac{\partial F_{1}^{(1)}}{\partial t}=\frac{\partial}{\partial t}\left\langle\delta\left(u^{(1)}-v^{(1)}\right) \delta\left(h^{(1)}-g^{(1)}\right) \delta\left(\theta^{(1)}-\phi^{(1)}\right)\right. \\
& \left.\delta\left(C^{(1)}-\psi^{(1)}\right)\right\rangle
\end{aligned}
$$$$
=\left\langle\delta\left(h^{(1)}-g^{(1)}\right) \delta\left(\theta^{(1)}-\phi^{(1)}\right) \delta\left(C^{(1)}-\psi^{(1)}\right)\right.
$$$$
\left.\frac{\partial}{\partial t} \delta\left(u^{(1)}-v^{(1)}\right)\right\rangle+\left\langle\delta\left(u^{(1)}-v^{(1)}\right) \delta\left(\theta^{(1)}-\phi^{(1)}\right)\right.
$$$$
\left.\times \delta\left(C^{(1)}-\psi^{(1)}\right) \frac{\partial}{\partial t} \delta\left(h^{(1)}-g^{(1)}\right)\right\rangle
$$$$
+\left\langle\delta\left(u^{(1)}-v^{(1)}\right) \delta\left(h^{(1)}-g^{(1)}\right) \delta\left(\theta^{(1)}-\phi^{(1)}\right)\right.
$$

$\left.\frac{\partial}{\partial t} \delta\left(C^{(1)}-\psi^{(1)}\right)\right\rangle$ 
$=\left\langle-\delta\left(h^{(1)}-g^{(1)}\right) \delta\left(\theta^{(1)}-\phi^{(1)}\right) \delta\left(C^{(1)}-\psi^{(1)}\right)\right.$

$\left.\frac{\partial u^{(1)}}{\partial t} \frac{\partial}{\partial v^{(1)}} \delta\left(u^{(1)}-v^{(1)}\right)\right\rangle$

$+\left\langle-\delta\left(u^{(1)}-v^{(1)}\right) \delta\left(\theta^{(1)}-\phi^{(1)}\right) \delta\left(C^{(1)}-\psi^{(1)}\right)\right.$

$\left.\frac{\partial h^{(1)}}{\partial t} \frac{\partial}{\partial g^{(1)}} \delta\left(h^{(1)}-g^{(1)}\right)\right\rangle$

$+\left\langle-\delta\left(u^{(1)}-v^{(1)}\right) \delta\left(h^{(1)}-g^{(1)}\right) \delta\left(C^{(1)}-\psi^{(1)}\right)\right.$

$\left.\frac{\partial \theta^{(1)}}{\partial t} \frac{\partial}{\partial \phi^{(1)}} \delta\left(\theta^{(1)}-\phi^{(1)}\right)\right\rangle$

$+\left\langle-\delta\left(u^{(1)}-v^{(1)}\right) \delta\left(h^{(1)}-g^{(1)}\right) \delta\left(\theta^{(1)}-\phi^{(1)}\right)\right.$

$\left.\frac{\partial c^{(1)}}{\partial t} \frac{\partial}{\partial \psi^{(1)}} \delta\left(C^{(1)}-\psi^{(1)}\right)\right\rangle$

Using equations (8) - (11) in the equation (23), we get

$\frac{\partial F_{1}^{(1)}}{\partial t}=\left\langle-\delta\left(h^{(1)}-g^{(1)}\right) \delta\left(\theta^{(1)}-\phi^{(1)}\right) \delta\left(C^{(1)}-\psi^{(1)}\right)\right.$

$\left\{-\frac{\partial}{\partial x_{\beta}}\left(u_{\alpha}^{(1)} u_{\beta}^{(1)}-h_{\alpha}^{(1)} h_{\beta}^{(1)}\right)-\frac{1}{4 \pi} \frac{\partial}{\partial x_{\alpha}}\right\}$

$\left[\frac{\partial u_{\alpha}^{(1)}}{\partial x_{\beta}^{(1)}} \frac{\partial u_{\beta}^{(1)}}{\partial x_{\alpha}^{(1)}}-\frac{\partial h_{\alpha}^{(1)}}{\partial x_{\beta}^{(1)}} \frac{\partial h_{\beta}^{(1)}}{\partial x_{\alpha}^{(1)}}\right] \frac{d \bar{x}^{\prime}}{\left|\bar{x}^{\prime}-\bar{x}\right|}$

$\left.+\nu \nabla^{2} u_{\alpha}^{(1)}-2 \epsilon_{m \alpha \beta} \Omega_{m} u_{\alpha}^{(1)}\right\} \times \frac{\partial}{\partial v_{\alpha}^{(1)}}$,

$\left.\delta\left(u^{(1)}-v^{(1)}\right)\right\rangle+\left\langle-\delta\left(u^{(1)}-v^{(1)}\right) \delta\left(\theta^{(1)}-\phi^{(1)}\right)\right.$

$\delta\left(C^{(1)}-\psi^{(1)}\right)\left\{-\frac{\partial}{\partial x_{\beta}^{(1)}}\left(h_{\alpha}^{(1)} u_{\beta}^{(1)}-u_{\alpha}^{(1)} h_{\beta}^{(1)}\right)\right.$

$\left.\left.+\lambda \nabla^{2} h_{\alpha}^{(1)}\right\} \frac{\partial}{\partial g_{\alpha}^{(1)}} \delta\left(h^{(1)}-g^{(1)}\right)\right\rangle+$

$$
\begin{aligned}
& \left\langle-\delta\left(u^{(1)}-v^{(1)}\right) \delta\left(h^{(1)}-g^{(1)}\right) \delta\left(C^{(1)}-\psi^{(1)}\right)\right. \\
& \left.\times\left\{-u_{\beta}^{(1)} \frac{\partial \theta^{(1)}}{\partial x_{\beta}}+\gamma^{2} \theta^{(1)}\right\} \times \frac{\partial}{\partial \phi^{(1)}} \delta\left(\theta^{(1)}-\phi^{(1)}\right)\right\rangle+
\end{aligned}
$$$$
\left\langle-\delta\left(u^{(1)}-v^{(1)}\right) \delta\left(h^{(1)}-g^{(1)}\right)\right.
$$$$
\times \delta\left(\theta^{(1)}-\phi^{(1)}\right)\left\{-u_{\beta}^{(1)} \frac{\partial c^{(1)}}{\partial x_{\beta}^{(1)}}+D \nabla^{2} C\right\}
$$$$
\left.\frac{\partial}{\partial \psi^{(1)}} \delta\left(C^{(1)}-\psi^{(1)}\right)\right\rangle
$$$$
=\left\langle\delta\left(h^{(1)}-g^{(1)}\right) \delta\left(\theta^{(1)}-\phi^{(1)}\right) \delta\left(C^{(1)}-\psi^{(1)}\right)\right.
$$$$
\left.\frac{\partial u_{\alpha}^{(1)} u_{\beta}^{(1)}}{\partial x_{\beta}^{(1)}} \frac{\partial}{\partial v_{\alpha}^{(1)}} \delta\left(u^{(1)}-v^{(1)}\right)\right\rangle
$$$$
+\left\langle-\delta\left(h^{(1)}-g^{(1)}\right) \delta\left(\theta^{(1)}-\phi^{(1)}\right) \delta\left(C^{(1)}-\psi^{(1)}\right)\right.
$$

$\left.\frac{\partial h_{\alpha}^{(1)} h_{\beta}^{(1)}}{\partial x_{\beta}^{(1)}} \frac{\partial}{\partial v_{\alpha}} \delta\left(u^{(1)}-v^{(1)}\right)\right\rangle$

$+\left\langle\delta\left(h^{(1)}-g^{(1)}\right) \delta\left(\theta^{(1)}-\phi^{(1)}\right) \delta\left(C^{(1)}-\psi^{(1)}\right)\right.$

$\frac{1}{4 \pi} \frac{\partial}{\partial x_{\alpha}^{(1)}} \int\left[\frac{\partial u_{\alpha}^{(1)}}{\partial x_{\beta}^{(1)}} \frac{\partial u_{\beta}^{(1)}}{\partial x_{\alpha}^{(1)}}-\frac{\partial h_{\alpha}^{(1)}}{\partial x_{\beta}^{(1)}} \frac{\partial h_{\beta}^{(1)}}{\partial x_{\alpha}^{(1)}}\right]$

$\left.\times \frac{d \bar{x}^{\prime}}{\left|\bar{x}^{\prime}-\bar{x}\right|} \frac{\partial}{\partial v_{\alpha}^{(1)}} \delta\left(u^{(1)}-v^{(1)}\right)\right\rangle+\left\langle-\delta\left(h^{(1)}-g^{(1)}\right)\right.$

$\left.\delta\left(\theta^{(1)}-\phi^{(1)}\right) \delta\left(C^{(1)}-\psi^{(1)}\right) \nu \nabla^{2} u_{\alpha}^{(1)} \frac{\partial}{\partial v_{\alpha}^{(1)}} \delta\left(u^{(1)}-v^{(1)}\right)\right\rangle$

$+\left\langle\delta\left(h^{(1)}-g^{(1)}\right) \delta\left(\theta^{(1)}-\phi^{(1)}\right) \delta\left(C^{(1)}-\psi^{(1)}\right)\right.$

$\left.2 \epsilon_{m \alpha \beta} \Omega_{m} u_{\alpha}^{(1)} \frac{\partial}{\partial v_{\alpha}^{(1)}} \delta\left(u^{(1)}-v^{(1)}\right)\right\rangle$

$+\left\langle\delta\left(u^{(1)}-v^{(1)}\right) \delta\left(\theta^{(1)}-\phi^{(1)}\right) \delta\left(C^{(1)}-\psi^{(1)}\right)\right.$

$\left.\frac{\partial h_{\alpha}^{(1)} u_{\beta}^{(1)}}{\partial x_{\beta}^{(1)}} \frac{\partial}{\partial g_{\alpha}^{(1)}} \delta\left(h^{(1)}-g^{(1)}\right)\right\rangle$ 
$+\left\langle-\delta\left(u^{(1)}-v^{(1)}\right) \delta\left(\theta^{(1)}-\phi^{(1)}\right) \delta\left(C^{(1)}-\psi^{(1)}\right)\right.$

$\left.\frac{\partial u_{\alpha}^{(1)} h_{\beta}^{(1)}}{\partial x_{\beta}^{(1)}} \frac{\partial}{\partial g_{\alpha}} \delta\left(h^{(1)}-g^{(1)}\right)\right\rangle$

$+\left\langle-\delta\left(u^{(1)}-v^{(1)}\right) \delta\left(\theta^{(1)}-\phi^{(1)}\right) \delta\left(C^{(1)}-\psi^{(1)}\right)\right.$.

$\left.\lambda \nabla^{2} h_{\alpha}^{(1)} \frac{\partial}{\partial g_{\alpha}^{(1)}} \delta\left(h^{(1)}-g^{(1)}\right)\right\rangle$

$+\left\langle\delta\left(u^{(1)}-v^{(1)}\right) \delta\left(h^{(1)}-g^{(1)}\right) \delta\left(C^{(1)}-\psi^{(1)}\right)\right.$

$\left.u_{\beta}^{(1)} \frac{\partial \theta^{(1)}}{\partial x_{\beta}^{(1)}} \frac{\partial}{\partial \phi^{(1)}} \delta\left(\theta^{(1)}-\phi^{(1)}\right)\right\rangle$

$+\left\langle-\delta\left(u^{(1)}-v^{(1)}\right) \delta\left(h^{(1)}-g^{(1)}\right) \delta\left(C^{(1)}-\psi^{(1)}\right)\right.$

$\left.\nabla^{2} \theta^{(1)} \frac{\partial}{\partial \phi^{(1)}} \delta\left(\theta^{(1)}-\phi^{(1)}\right)\right\rangle$

$+\left\langle\delta\left(u^{(1)}-v^{(1)}\right) \delta\left(h^{(1)}-g^{(1)}\right) \delta\left(\theta^{(1)}-\phi^{(1)}\right)\right.$

$\left.u_{\beta}^{(1)} \frac{\partial C^{(1)}}{\partial x_{\beta}^{(1)}} \frac{\partial}{\partial \psi^{(1)}} \delta\left(C^{(1)}-\psi^{(1)}\right)\right\rangle$

$+\left\langle-\delta\left(u^{(1)}-v^{(1)}\right) \delta\left(h^{(1)}-g^{(1)}\right) \delta\left(\theta^{(1)}-\phi^{(1)}\right)\right.$.

$\left.D \nabla^{2} C^{(1)} \frac{\partial}{\partial \psi^{(1)}} \delta\left(C^{(1)}-\psi^{(1)}\right)\right\rangle$

$+\left\langle-\delta\left(u^{(1)}-v^{(1)}\right) \delta\left(h^{(1)}-g^{(1)}\right) \delta\left(\theta^{(1)}-\phi^{(1)}\right)\right.$.

$\left.R C^{(1)} \frac{\partial}{\partial \psi^{(1)}} \delta\left(C^{(1)}-\psi^{(1)}\right)\right\rangle$

Various terms in the equation (24) can be simplified as that they may be expressed in terms of one point and two point distribution functions.

Simplifying each term of the equation (24), we get the transport equation for one point distribution function $F_{1}{ }^{(1)}(\mathrm{v}, \mathrm{g}, \phi$, $\psi$ ) in MHD turbulence for concentration undergoing a first order reaction in a rotating system as

$$
\begin{aligned}
& \frac{\partial F_{1}^{(1)}}{\partial t}+v_{\beta}^{(1)} \frac{\partial F_{1}^{(1)}}{\partial x_{\beta}^{(1)}}+g_{\beta}^{(1)}\left(\frac{\partial g_{\alpha}^{(1)}}{\partial v_{\alpha}^{(1)}}+\frac{\partial v_{\alpha}^{(1)}}{\partial g_{\alpha}^{(1)}}\right) \frac{\partial F_{1}^{(1)}}{\partial x_{\beta}^{(1)}}-\frac{\partial}{\partial v_{\alpha}^{(1)}} \\
& {\left[\frac{1}{4 \pi} \iiint \iint \frac{\partial}{\partial x_{\alpha}^{(1)}}\left(\frac{1}{\left|\bar{x}^{(2)}-\bar{x}^{(1)}\right|}\right)\right.} \\
& \times\left(\frac{\partial v_{\alpha}^{(2)}}{\partial x_{\beta}^{(2)}} \frac{\partial v_{\beta}^{(2)}}{\partial x_{\alpha}^{(2)}}-\frac{\partial g_{\alpha}^{(2)}}{\partial x_{\beta}^{(2)}} \frac{\partial g_{\beta}^{(2)}}{\partial x_{\alpha}^{(2)}}\right) \\
& \left.F_{2}^{(1,2)} d x^{(2)} d v^{(2)} d g^{(2)} d \phi^{(2)} d \psi^{(2)}\right] \\
& +v \frac{\partial}{\partial v_{\alpha}^{(1)}} \operatorname{Lim}_{\bar{x}^{(2)} \rightarrow \bar{x}^{(1)}} \frac{\partial^{2}}{\partial x_{\beta}^{(2)} \partial x_{\beta}^{(2)}} \\
& \iiint \int v_{\alpha}^{(2)} F_{2}^{(1,2)} d v^{(2)} d g^{(2)} d \phi^{(2)} d \psi^{(2)} \\
& +\lambda \frac{\partial}{\partial g_{\alpha}^{(1)}} \operatorname{Lim}_{\bar{x}^{(2)} \rightarrow \bar{x}^{(1)}} \frac{\partial^{2}}{\partial x_{\beta}^{(2)} \partial x_{\beta}^{(2)}} \\
& \iiint \int g_{\alpha}^{(2)} F_{2}^{(1,2)} d v^{(2)} d g^{(2)} d \phi^{(2)} d \psi^{(2)} \\
& +\gamma \frac{\partial}{\partial \phi^{(1)}} \operatorname{Lim}_{\bar{x}^{(2)} \rightarrow \bar{x}^{(1)}} \frac{\partial^{2}}{\partial x_{\beta}^{(2)} \partial x_{\beta}^{(2)}} \\
& \iiint \int \phi^{(2)} F_{2}^{(1,2)} d v^{(2)} d g^{(2)} d \phi^{(2)} d \psi^{(2)} \\
& +D \frac{\partial}{\partial \psi^{(1)}} \operatorname{Lim}_{\bar{x}^{(2)} \rightarrow \bar{x}^{(1)}} \frac{\partial^{2}}{\partial x_{\beta}^{(2)} \partial x_{\beta}^{(2)}} \\
& \iiint \int \psi^{(2)} F_{2}^{(1,2)} d v^{(2)} d g^{(2)} d \phi^{(2)} d \psi^{(2)} \\
& +2 \epsilon_{m \alpha \beta} \Omega_{m} F_{1}^{(1)}-R \psi^{(1)} \frac{\partial}{\partial \psi^{(1)}} F_{1}^{(1)}=0
\end{aligned}
$$

Similarly, an equation for two-point distribution function $\mathrm{F}_{2}{ }^{(1,2)}$ in MHD turbulence for concentration undergoing a first order reaction in a rotating system can be derived by differentiating equation (13) and using equations (2), (3),(4),(8) and simplifying in the same manner, which is 


$$
\begin{array}{ll}
\frac{\partial F_{2}^{(1,2)}}{\partial t}+\left(v_{\beta}^{(1)} \frac{\partial}{\partial x_{\beta}^{(1)}}+v_{\beta}^{(2)} \frac{\partial}{\partial x_{\beta}^{(2)}}\right) F_{2}^{(1,2)}+ & +D\left(\frac{\partial}{\partial \psi^{(1)}} \operatorname{Lim}_{\bar{x}^{(3)} \rightarrow \bar{x}^{(1)}}+\frac{\partial}{\partial \psi^{(2)}} \operatorname{Lim}_{\bar{x}^{(3)} \rightarrow \bar{x}^{(2)}}\right) \frac{\partial^{2}}{\partial x_{\beta}^{(3)} \partial x_{\beta}^{(3)}} \\
g_{\beta}^{(1)}\left(\frac{\partial g_{\alpha}^{(1)}}{\partial v_{\alpha}^{(1)}}+\frac{\partial v_{\alpha}^{(1)}}{\partial g_{\alpha}^{(1)}}\right) \frac{\partial}{\partial x_{\beta}^{(1)}} F_{2}^{(1,2)} & \iiint \int \psi^{(3)} F_{3}^{(1,2,3)} d v^{(3)} d g^{(3)} d \phi^{(3)} d \psi^{(3)} \\
+g_{\beta}^{(2)}\left(\frac{\partial g_{\alpha}^{(2)}}{\partial v_{\alpha}^{(2)}}+\frac{\partial v_{\alpha}^{(2)}}{\partial g_{\alpha}^{(2)}}\right) \frac{\partial}{\partial x_{\beta}^{(2)}} F_{2}^{(1,3)}-\frac{\partial}{\partial v_{\alpha}^{(1)}} & +2 \in_{m \alpha \beta} \Omega_{m} F_{2}^{(1,2)}-R \psi^{(1)} \frac{\partial}{\partial \psi^{(1)}} F_{1}^{(1)}=0
\end{array}
$$$$
\left[\frac{1}{4 \pi} \iiint \iint \frac{\partial}{\partial x_{\alpha}^{(1)}}\left(\frac{1}{\left|\bar{x}^{(3)}-\bar{x}^{(1)}\right|}\right)\right.
$$$$
\times\left(\frac{\partial v_{\alpha}^{(3)}}{\partial x_{\beta}^{(3)}} \frac{\partial v_{\beta}^{(3)}}{\partial x_{\alpha}^{(3)}}-\frac{\partial g_{\alpha}^{(3)}}{\partial x_{\beta}^{(3)}} \frac{\partial g_{\beta}^{(3)}}{\partial x_{\alpha}^{(3)}}\right)
$$$$
\left.F_{3}^{(1,2,3)} d x^{(3)} d v^{(3)} d g^{(3)} d \phi^{(3)} d \psi^{3)}\right]
$$$$
-\frac{\partial}{\partial v_{\alpha}^{(2)}}\left[\frac{1}{4 \pi} \iiint \iint \frac{\partial}{\partial x_{\alpha}^{(2)}}\left(\frac{1}{\left|\bar{x}^{(3)}-\bar{x}^{(2)}\right|}\right)\right.
$$$$
\left(\frac{\partial v_{\alpha}^{(3)}}{\partial x_{\beta}^{(3)}} \frac{\partial v_{\beta}^{(3)}}{\partial x_{\alpha}^{(3)}}-\frac{\partial g_{\alpha}^{(3)}}{\partial x_{\beta}^{(3)}} \frac{\partial g_{\beta}^{(3)}}{\partial x_{\alpha}^{(3)}}\right)
$$$$
\left.\times F_{3}^{(1,2,3)} d x^{(3)} d v^{(3)} d g^{(3)} d \phi^{(3)} d \psi^{3)}\right]
$$$$
+v\left(\frac{\partial}{\partial v_{\alpha}^{(1)}} \operatorname{Lim}_{\bar{x}^{(3)} \rightarrow \bar{x}^{(1)}}+\frac{\partial}{\partial v_{\alpha}^{(2)}} \operatorname{Lim}_{\bar{x}^{(3)} \rightarrow \bar{x}^{(2)}}\right)
$$$$
\frac{\partial^{2}}{\partial x_{\beta}^{(3)} \partial x_{\beta}^{(3)}} \iiint \int v_{\alpha}^{(3)} F_{3}^{(1,2,3)} d v^{(3)} d g^{(3)} d \phi^{(3)} d \psi^{(3)}
$$

Following this way, we can derive the equations for evolution of $F_{3}^{(1,2,3)}, F_{4}{ }^{(1,2,3,4)}$ and so on. Logically, it is possible to have an equation for every Fn ( $\mathrm{n}$ is an integer) but the system of equations so obtained is not closed. It seems that certain approximations will be required thus obtained.

\section{Results and Discussion}

If the system is non rotating then $\Omega \mathrm{m}=0$, the transport equation for one point distribution function in MHD turbulent flow (25) becomes

$$
\begin{aligned}
& \frac{\partial F_{1}^{(1)}}{\partial t}+v_{\beta}^{(1)} \frac{\partial F_{1}^{(1)}}{\partial x_{\beta}^{(1)}}+g_{\beta}^{(1)}\left(\frac{\partial g_{\alpha}^{(1)}}{\partial v_{\alpha}^{(1)}}+\frac{\partial v_{\alpha}^{(1)}}{\partial g_{\alpha}^{(1)}}\right) \frac{\partial F_{1}^{(1)}}{\partial x_{\beta}^{(1)}} \\
& -\frac{\partial}{\partial v_{\alpha}^{(1)}}\left[\frac{1}{4 \pi} \iiint \iint\left(\frac{\partial}{\partial x_{\alpha}^{(1)}} \frac{1}{\left|\bar{x}^{(2)}-\bar{x}^{(1)}\right|}\right)\right. \\
& \times\left(\frac{\partial v_{\alpha}^{(2)}}{\partial x_{\beta}^{(2)}} \frac{\partial v_{\beta}^{(2)}}{\partial x_{\alpha}^{(2)}}-\frac{\partial g_{\alpha}^{(2)}}{\partial x_{\beta}^{(2)}} \frac{\partial g_{\beta}^{(2)}}{\partial x_{\alpha}^{(2)}}\right) \\
& F_{2}^{(1,2)} d x^{(2)} d v^{(2)} d g^{(2)} d \phi^{(2)} d \psi^{(2)} \\
& +v \frac{\partial}{\partial v_{\alpha}^{(1)}} \operatorname{Lim}_{\bar{x}^{(2)} \rightarrow \bar{x}^{(1)}} \frac{\partial^{2}}{\partial x_{\beta}^{(2)} \partial x_{\beta}^{(2)}} \\
& \iiint \int v_{\alpha}^{(2)} F_{2}^{(1,2)} d v^{(2)} d g^{(2)} d \phi^{(2)} d \psi^{(2)} \\
& +\gamma\left(\frac{\partial}{\partial \phi^{(1)}} \operatorname{Lim}_{\bar{x}^{(3)} \rightarrow \bar{x}^{(1)}}+\frac{\partial}{\partial \phi^{(2)}} \operatorname{Lim}_{\bar{x}^{(3)} \rightarrow \bar{x}^{(2)}}\right) \frac{\partial^{2}}{\partial x_{\beta}^{(3)} \partial x_{\beta}^{(3)}} \cdot \begin{array}{l}
+\lambda \frac{\partial}{\partial g_{\alpha}^{(1)}} \operatorname{Lim}_{\bar{x}^{(2)} \rightarrow \bar{x}^{(1)}} \frac{\partial^{2}}{\partial x_{\beta}^{(2)} \partial x_{\beta}^{(2)}} \\
\iiint \int g_{\alpha}^{(2)} F_{2}^{(1,2)} d v^{(2)} d g^{(2)} d \phi^{(2)} d \psi^{(2)}
\end{array}
\end{aligned}
$$$$
\frac{\partial^{2}}{\partial x_{\beta}^{(3)} \partial x_{\beta}^{(3)}} \iiint \int g_{\alpha}^{(3)} F_{3}^{(1,2,3)} d v^{(3)} d g^{(3)} d \phi^{3)} d \psi^{(3)}
$$$$
\iiint \int \phi^{(3)} F_{3}^{(1,2,3)} d v^{(3)} d g^{(3)} d \phi^{(3)} d \psi^{(3)}
$$ 


$$
\begin{aligned}
& +\gamma \frac{\partial}{\partial \phi^{(1)}} \operatorname{Lim}_{\bar{x}^{(2)} \rightarrow \bar{x}^{(1)}} \frac{\partial^{2}}{\partial x_{\beta}^{(2)} \partial x_{\beta}^{(2)}} \\
& \iiint \int \phi^{(2)} F_{2}^{(1,2)} d v^{(2)} d g^{(2)} d \phi^{(2)} d \psi^{(2)} \\
& +D \frac{\partial}{\partial \psi^{(1)}} \operatorname{Lim}_{\bar{x}^{(2)} \rightarrow \bar{x}^{(1)}} \frac{\partial^{2}}{\partial x_{\beta}^{(2)} \partial x_{\beta}^{(2)}} \iiint \int \psi^{(2)} F_{2}^{(1,2)} d v^{(2)} \\
& d g^{(2)} d \phi^{(2)} d \psi^{(2)}-R \psi^{(1)} \frac{O}{\partial \psi^{(1)}} F_{1}^{(1)}=0
\end{aligned}
$$

which was obtained earlier by (Sarker and Islam, 2007).

We can exhibit an analogy of this equation with the first equation in BBGKY hierarchy in the kinetic theory of gases. The first equation of BBGKY hierarchy is given as

$$
\begin{aligned}
& \frac{\partial F_{1}^{(1)}}{\partial t}+\frac{1}{m} v_{\beta}^{(1)} \frac{\partial}{\partial x_{\beta}^{(1)}} F_{1}^{(1)} \\
& =n \iint \frac{\partial \psi_{1,2}}{\partial x_{\alpha}^{(1)}} \frac{\partial F_{2}^{(1,2)}}{\partial v_{\alpha}^{(1)}} d \bar{x}^{(2)} d \bar{v}^{(2)}
\end{aligned}
$$

where $\psi_{1.2}=\psi\left|v_{\alpha}^{(2)}-v_{\alpha}^{(1)}\right|$ is the inter molecular potential.

If we drop the viscous, magnetic and thermal diffusive, concentration terms and constant reaction terms from the one point evolution equation (28), we have

$$
\begin{aligned}
& \frac{\partial F_{1}^{(1)}}{\partial t}+v_{\beta}^{(1)} \frac{\partial F_{1}^{(1)}}{\partial x_{\beta}^{(1)}}+g_{\beta}^{(1)}\left(\frac{\partial g_{\alpha}^{(1)}}{\partial v_{\alpha}^{(1)}}+\frac{\partial v_{\alpha}^{(1)}}{\partial g_{\alpha}^{(1)}}\right) \\
& \frac{\partial F_{1}^{(1)}}{\partial x_{\beta}^{(1)}}-\frac{\partial}{\partial v_{\alpha}^{(1)}}\left[\frac{1}{4 \pi} \iiint \iint \frac{\partial}{\partial x_{\alpha}^{(1)}}\left(\frac{1}{\left|\bar{x}^{(2)}-\bar{x}^{(1)}\right|}\right)\right. \\
& \times\left(\frac{\partial v_{\alpha}^{(2)}}{\partial x_{\beta}^{(2)}} \frac{\partial v_{\beta}^{(2)}}{\partial x_{\alpha}^{(2)}}-\frac{\partial g_{\alpha}^{(2)}}{\partial x_{\beta}^{(2)}} \frac{\partial g_{\beta}^{(2)}}{\partial x_{\alpha}^{(2)}}\right) \\
& F_{2}^{(1,2)} d x^{(2)} d v^{(2)} d g^{(2)} d \phi^{(2)} d \psi^{(2)} \mid=0 \\
& \text { The existence of the term } \frac{\partial g_{\alpha}^{(1)}}{\partial v_{\alpha}^{(1)}}+\frac{\partial v_{\alpha}^{(1)}}{\partial g_{\alpha}^{(1)}}
\end{aligned}
$$

can be explained on the basis that two characteristics of the flow field are related to each other and describe the interac- tion between the two modes (velocity and magnetic) at a single point $\mathrm{x}^{(1)}$.

The transport equation for distribution function of velocity, magnetic, temperature, concentration and reaction have been shown here to provide an advantageous basis for modeling the turbulent flows in a rotating system. Here we have made an attempt for the modeling of various terms such as fluctuating pressure, viscosity and diffusivity in order to close the equation for distribution function of velocity, magnetic, temperature, concentration and reaction. It is also possible to construct such type of distribution functions in variable density follows. The advantage of constructing such type hierarchy is to provide simultaneous information about velocity, magnetic temperature, concentration and reaction without knowledge of scale of turbulence.

\section{References}

Azad M. A. K and Sarker M. S. A (2003). Decay of MHD turbulence before the final period for the case of multipoint and multi-time in presence of dust particle. The Bangladesh Jour. of Sci. and Industrial Res. 38(3-4): 151-164

Dixit T. and Upadhyay B. N. (1989a). The effect of Coriolis force on acceleration covariance in MHD turbulent dusty flow with rotational symmetry. Astrophysics and Space Sci. 153: 257-268.

Kishore N. (1978). distribution functions in the statistical theory of MHD turbulence of an incompressible fluid J. Scientific Res., B. H. U., 28(2): 163.

Kishore N. and Singh S. R. (1984). Transport equation for the bivariate joint distribution function of velocity and temperature in turbulent flow. Bull. Tech. Univ., Istambul, 37: 91.

Kishore N. and Singh S. R. (1984). The effect of Coriolis force on acceleration covariance in turbulent flow with rotational symmetry. Astrophysics and Space Sci., 104: 121-125

Kishore N. and Singh S. R. (1985). The transport equation for the joint distribution function of velocity, temperature and concentration in convective turbulent flow. Prog. of Maths, B. H. U., 19(1 and 2): 13. 
Kollman W. and Janica J. (1982). The transport equation for the probability density function of a scalar in turbulent shear flow. Physics of Fluids, 25: 1955.

Lundgren T. S. (1967). Hierarchy of coupled equations for multi-point turbulence velocity distribution functions. Physics of Fluids, 10: 967.

Pope S. B. (1981b). The transport equation for the joint probability density function of velocity and scalars in turbulent flow. Physics of Fluid, 24: 588.

Sarker M. S. A.and Kishore N.(1991a). Distribution functions in the statistical theory of convective MHD turbulence of an incompressible fluid. Astrophysics and Space Sci.,181,29.

Sarker M. S. A. and Kishore N. (1991b): Decay of MHD turbulence before the final period. Int. J. Engng. Sci., 29: 1479-1485.

Sarker M. S. A.and Kishore N. (1999): Distribution functions in the statistical theory of convective MHD turbulence of mixture of a miscible incompressible fluid Prog of Math., B.H.U. India, 33(1and 2): 83.
Ta-You Wu (1966). Kinetic Theory of Gases and Plasma, Addision Wesley Phlelishing Company.

Sarker M. S. A and Islam M. A (2001) Decay of MHD turbulence before the final period for the case of multipoint and multi-time. Indian J. Pure and Appl. Math.32(7): 1065-1076.

Sarker M. S. A. and Islam M. A. (2002). Distribution functions in the statistical theory of convective MHD turbulence of an incompressible fluid in a rotating system. Rajshahi university studies. Part-B. J. Sci. 30.

Islam M. A and Sarker M. S. A. (2007): Distribution functions in the statistical theory of MHD turbulence for velocity and concentration undergoing a first order reaction. Rajshahi university studies. Part-B. J. Sci. 35.

Received : November 09, 2009;

Accepted : August 04, 2010 